\title{
Effects of thermal water on skin regeneration
}

\author{
ANGELA FAGA $^{1}$, GIOVANNI NICOLETTI $^{1}$, CESARINA GREGOTTI $^{3}$, VALENTINA FINOTTI $^{1}$, \\ AGNESE NITTO $^{1}$ and LUCIANA GIOGLIO ${ }^{2}$
}

\author{
${ }^{1}$ Department of Plastic Surgery, University of Pavia - IRCCS Fondazione 'S. Maugeri'; ${ }^{2}$ Department of Human Anatomy, \\ University of Pavia; ${ }^{3}$ Department of Cellular and Molecular Pharmacology, University of Pavia, Pavia, Italy
}

Received October 26, 2011; Accepted December 8, 2011

DOI: $10.3892 / \mathrm{ijmm} .2012 .917$

\begin{abstract}
An experimental study was carried out in an animal (New Zealand white rabbit) wound model to evaluate any effects of a hypotonic, bicarbonate-calcium-magnesium mineral water (Comano thermal water) on skin regeneration, comparing the healing rate of split-thickness skin graft donor sites treated with the thermal water wet dressing versus a standard petrolatum gauze dressing versus a saline solution wet dressing. The study was performed in two steps; an overall of 22 animals were enrolled in the study. The wound healing progress was evaluated both by the surgeons and by the histologists. Sixty-four punch biopsies were examined in all. The histological samples were examined after staining with haematoxylin and eosin, Masson's and orcein staining and under a transmission electron microscope. The data were statistically analysed. The Comano thermal water proved to improve skin regeneration, not only by increasing keratinocyte proliferation and migration but also favourably modulating the regenerated collagen and elastic fibres in the dermis. We propose that the results of the topical treatment with the thermal water could be due to the favourable combination of a local wet environment with an anti-inflammatory action and that the regenerative properties of Comano thermal water observed in rabbits could also be applied for human use.
\end{abstract}

\section{Introduction}

The Comano thermal water is a hypotonic, bicarbonatecalcium-magnesium mineral water which is rich in fluoride, has a neutral $\mathrm{pH}$ and a low buffer capacity. It has a well-recognised anti-inflammatory action and is successfully used in the treatment of different skin diseases, such as psoriasis and atopic dermatitis (1-3).

An experimental study was carried out at the Plastic and Reconstructive Surgery Unit of the University of Pavia in

Correspondence to: Professor Angela Faga, Department of Plastic Surgery, University of Pavia - IRCCS Fondazione 'S Maugeri', Via S. Maugeri 10, I-27100 Pavia, Italy

E-mail: angela.faga@unipv.it

Key words: thermal water, re-epithelialisation, dermis modulation order to evaluate the effects of the Comano thermal water in skin regeneration.

In an experimental animal wound model we compared the healing rate of split-thickness skin graft donor sites treated with the Comano thermal water wet dressing versus a standard petrolatum gauze dressing versus a saline solution wet dressing.

\section{Materials and methods}

The trial was approved by the Ethics Committee of the University of Pavia on June 3rd, 2008. The study was performed in two steps in an experimental animal model, according to human treatment of animals, in the animal breeding department of the University of Pavia.

First step. The aim of the first step of the study was the demonstration of any favourable effect of the Comano thermal water on the wound healing process in comparison with the other treatments and the close examination of key timing details of its action. Six New Zealand white rabbits were enrolled in the study and numbered from 1 to 6 . The animals were all male and had a mean weight of $1.5-2 \mathrm{~kg}$.

The experimental procedure was scheduled along 7 consecutive days, from Day 0 to 6. On Day 0, after shaving with an electric shaver, three $4-\mathrm{cm}^{2}$ areas (A, B and C) were marked on the skin of the back of each rabbit. General anaesthesia was induced through injection of $0.5 \mathrm{ml} / \mathrm{kg}$ of sodium thiopental into the marginal vein of the ear.

Split thickness skin grafts were harvested by the same investigator from the previously marked areas (A, B and C) with a Silver free-hand dermatome (Fig. 1). Each split thickness skin graft donor site was covered with a monolayer petrolatum gauze, two layers of sterile non-woven fabric gauze and a transparent film dressing (Tegaderm ${ }^{\circledR}$ ) (Fig. 2).

No further treatment was applied to area A. The gauzes on area B were soaked with $2 \mathrm{ml}$ of sterile saline solution with a 21-G needle drilling through the Tegaderm ${ }^{\circledR}$ film. The gauzes on area $\mathrm{C}$ were soaked with the same amount of sterile Comano thermal water in the same way. In order to prevent dressings removal, the rabbits were wrapped around with a tubular net bandage and a custom-made close-fitting coat secured with tight laces (Fig. 3).

After $24 \mathrm{~h}$ (Day 1), following local infiltration of 2\% lidocaine, a punch biopsy for histopathological examination was harvested in rabbit 1 from each of the treated areas, A, B and 


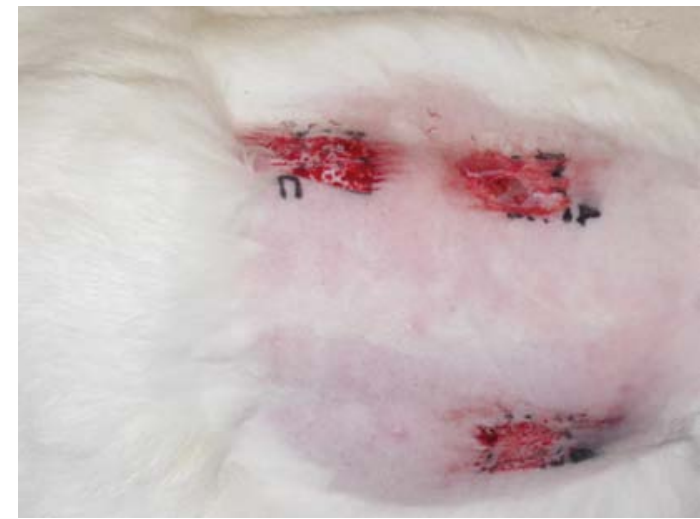

Figure 1. Back of an experimental rabbit after harvesting of split thickness skin grafts.

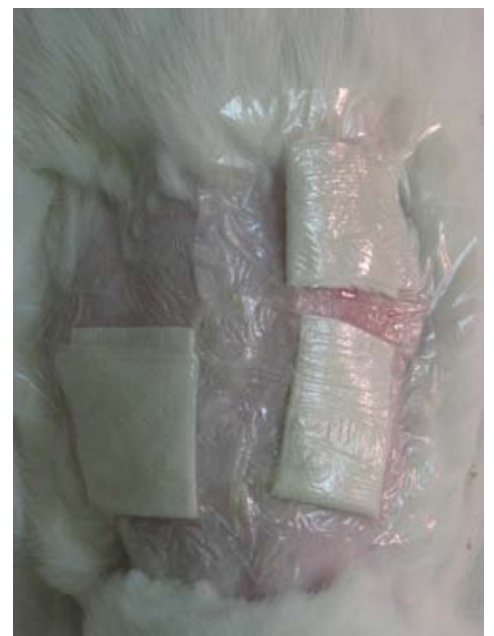

Figure 2. Dressings of the donor sites. The dressing of area $\mathrm{B}$ is going to be soaked with sterile normal saline; the dressing of area $\mathrm{C}$ is going to be soaked with sterile Comano water.

$\mathrm{C}$, and from an area of neighbouring normal skin. The animal was then sacrificed according to ethical guidelines.

The other 5 rabbits were treated repeating the soaking of the dressings of areas $\mathrm{B}$ and $\mathrm{C}$ with sterile saline solution and sterile Comano thermal water, respectively, using the same procedure of Day 0. On Day 2, punch biopsies were harvested in rabbit 2 which was then sacrificed, and the soaking procedure in the areas A and B was repeated in the remaining four rabbits. On Days 3 to 6 the above-mentioned schedule of one animal being sacrificed per day after punch biopsy and soaking renewal in the remaining rabbits was carried out.

Histological examinations were processed in the laboratories of the Department of Human Anatomy of the University of Pavia. The samples were fixed in $10 \%$ neutral buffered formalin, dehydrated with gradient alcohol series, cleared in xylene and eventually embedded in paraffin. Serial sections (5 $\mu \mathrm{m})$ were obtained using a Leitz (Wetzlar Germany) microtome, stained with haematoxylin and eosin (H\&E) and examined with a Nikon (Tokyo, Japan) Eclipse 80i microscope.

An overall of 24 punch biopsies were examined by the histologist. The regenerated epithelial cover features and the

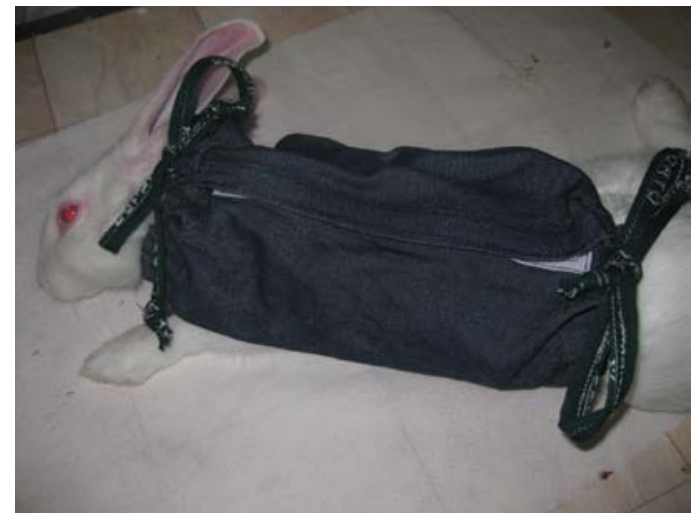

Figure 3. A rabbit wearing a close-fitting coat with tight laces to prevent dressings removal.

Table I. Semi-quantitative score scales used for the assessment of the regenerated epithelial layer features (scale 1) and the amount of the inflammatory infiltrate (scale 2).

\begin{tabular}{ll}
\hline Score & \multicolumn{1}{c}{ Description } \\
\hline Scale 1 & No epithelial cells \\
0 & $\begin{array}{l}\text { Early signs of re-epithelialisation } \\
\text { (scattered epithelial cells) }\end{array}$ \\
1 & Regenerated mono-layered epithelium \\
2 & Regenerated multi-layered epithelium \\
3 & \\
Scale 2 & No inflammatory infiltrate \\
0 & Few white cells \\
1 & Fair inflammatory infiltrate \\
2 & Rich inflammatory infiltrate \\
3 &
\end{tabular}

amount of the inflammatory infiltrate were assessed using a semi-quantitative scale, with scores ranging from 0 to 3 (Table I).

The Wilcoxon signed rank test was used for the comparative analysis of score distributions at different times. Variations in the epithelialisation score and the inflammatory infiltrate score were respectively considered positive and negative.

Second step. As in the first step of the trial the greatest differences among the effects on healing of different dressings were observed on Days 3 and 4, we thus decided to repeat the experience in a larger sample of rabbits, harvesting biopsies only on Days 3 and 4. Sixteen New Zealand white rabbits were enrolled in the study and numbered from 1 to 16 . As in the first step of the study, the animals were all male and had a mean weight of $1.5-2 \mathrm{~kg}$.

The experimental procedure was scheduled along 5 consecutive days, from Days 0 to 4. On Day 0, as in the first step of the study, split skin grafts were harvested from three different areas on the back of each animal (A, B and C) and the donor sites were treated with different dressings: standard paraffin gauze dressing (area A), sterile saline solution wet dressing (area B) and sterile Comano thermal water wet dressing (area C). 

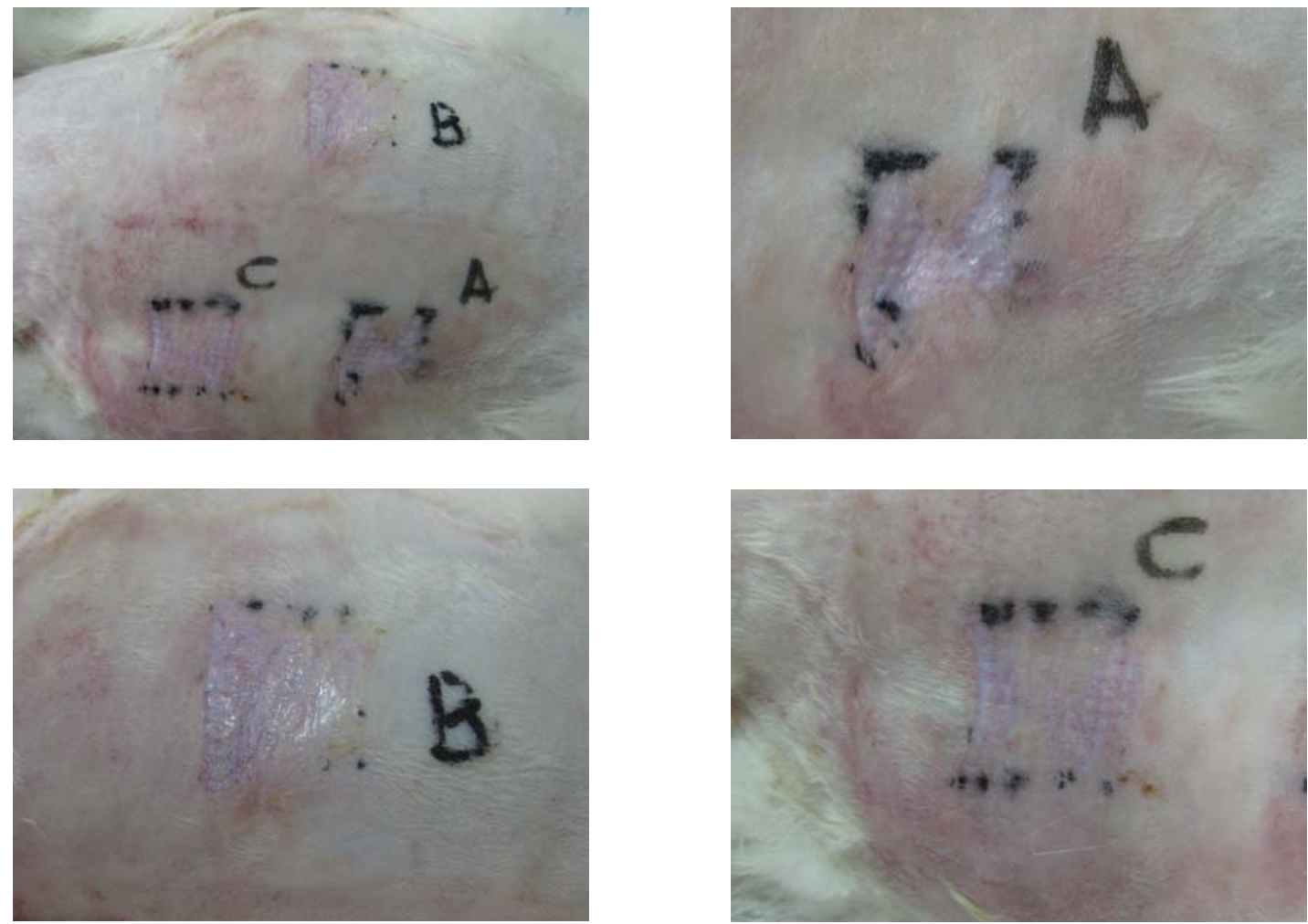

Figure 4. Back of an experimental rabbit 4 days after harvesting of split thickness skin grafts. Some new epithelium is detectable in all the areas. In a close-up of area A (petrolatum dressing) a fibrinous exudate is observable in more than half of the wound. In a close-up of area B (sterile saline solution dressing) the area looks slightly exudated. In a close-up of area C (sterile Comano water dressing) the area looks completely healed, pink and no exudate is observed.

On Days 1 and 2 wetting in the areas $\mathrm{B}$ and $\mathrm{C}$ was renewed with $2 \mathrm{ml}$ of sterile saline solution and sterile Comano thermal water, respectively. On Day 3, after local infiltration of $2 \%$ lidocaine, a punch biopsy was harvested in rabbits 1 to 8 from each of the treated areas A, B and C and from an area of neighbouring normal skin. The animals were then sacrificed. The other 8 rabbits were treated repeating the soaking of the dressings of areas B and C with sterile saline solution and sterile Comano thermal water, respectively. On Day 4, the same procedure of Day 3 (punch biopsies plus sacrifice) was performed in rabbits 9 to 16 .

These samples were all processed in the laboratories of the Department of Human Anatomy of the University of Pavia, according to the same procedures described in step 1. Besides conventional staining with H\&E, Masson's trichrome and orcein staining were performed as well, in order to investigate any significant pattern of collagen and elastic fibres.

The samples were also processed for an ultrastructural analysis. After fixing in $4 \%$ glutaraldehyde, they were dissolved in Millonig's phosphate buffer at $\mathrm{pH} 7.0$, for $4 \mathrm{~h}$ at $4^{\circ} \mathrm{C}$, washed in Millonig's phosphate buffer and post-fixed in a $1 \%$ osmium tetroxide solution in Millonig's phosphate buffer for $1 \mathrm{~h}$ at $4^{\circ} \mathrm{C}$. Then the specimens were dehydrated in alcohol gradients, treated with propyleneoxide, embedded in Epon 812 and cut into semi-thin and ultrathin sections using a RMC MT-X ultramicrotome (Scientific Corp., Tucson, AZ). Semithin sections for light microscopy were stained with toluidine blue. Ultrathin sections were stained with uranyl acetate and lead citrate, and examined with a Zeiss 109 (Zeiss, Germany) electron microscope.
An overall of 64 punch biopsies were examined by two independent observers. The regenerated epithelial layer features and the amount of the inflammatory infiltrate were assessed in the slices conventionally stained with H\&E, using the same semi-quantitative scale, with scores ranging from 0 to 3 , as in step 1 of the study. Similarly, the Wilcoxon signed rank test was used for the comparative analysis of score distributions at different times and variations in the epithelialisation score and the inflammatory infiltrate score were respectively considered positive and negative. Statistical analysis was carried out using the R program (4) while the Orange program (www.ailab.si/ orange) was used to elaborate the statistical charts.

\section{Results}

First step. Clinically, some interesting differences in the wound healing progress among the differently treated areas could be observed on Days 3 and 4. Particularly, on Day 4 in the area treated with petrolatum gauze dressing alone less than half of the wound looked healed, while elsewhere a fibrinous exudate was observable; also in the area treated with sterile saline solution just some spots and stripes looked healed while the others were slightly exudated and sunken; the area treated with sterile Comano water looked completely healed, pink in colour and without any exudates (Fig. 4).

Upon histological observation, differences in the wound healing progress among the differently treated areas along the trial are particularly evident on Days 3 and 4 (Table II, Fig. 5). Actually, on Day 2 only the area treated with Comano thermal water showed the early signs of healing; such signs were detect- 

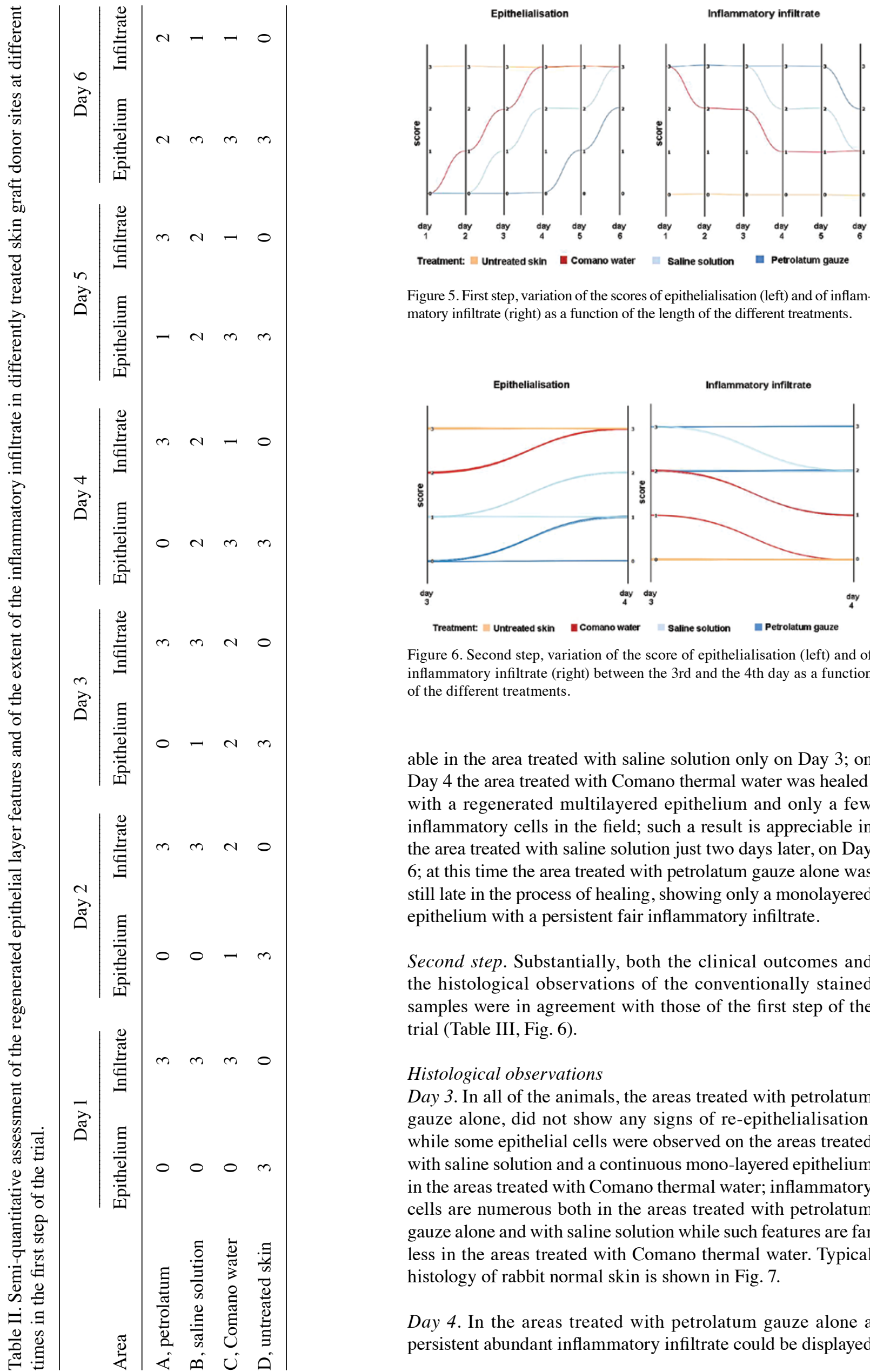

Figure 5. First step, variation of the scores of epithelialisation (left) and of inflammatory infiltrate (right) as a function of the length of the different treatments.

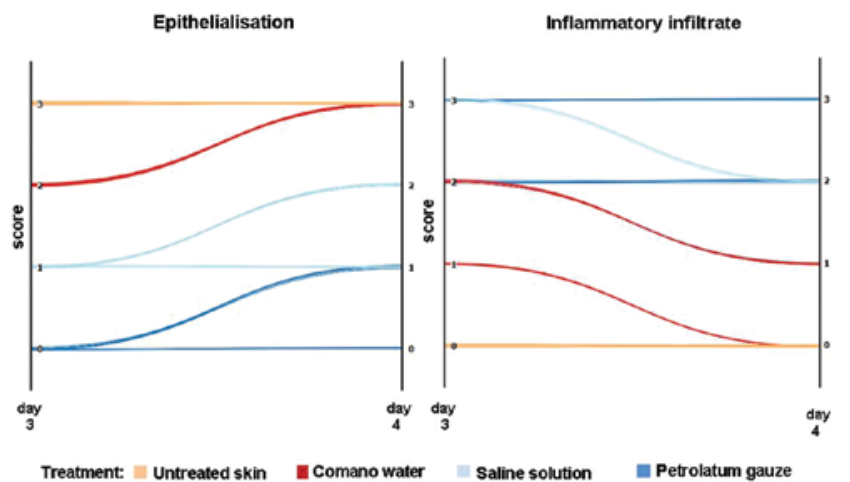

Figure 6. Second step, variation of the score of epithelialisation (left) and of inflammatory infiltrate (right) between the 3rd and the 4th day as a function of the different treatments.

able in the area treated with saline solution only on Day 3; on Day 4 the area treated with Comano thermal water was healed, with a regenerated multilayered epithelium and only a few inflammatory cells in the field; such a result is appreciable in the area treated with saline solution just two days later, on Day 6 ; at this time the area treated with petrolatum gauze alone was still late in the process of healing, showing only a monolayered epithelium with a persistent fair inflammatory infiltrate.

Second step. Substantially, both the clinical outcomes and the histological observations of the conventionally stained samples were in agreement with those of the first step of the trial (Table III, Fig. 6).

\section{Histological observations}

Day 3. In all of the animals, the areas treated with petrolatum gauze alone, did not show any signs of re-epithelialisation, while some epithelial cells were observed on the areas treated with saline solution and a continuous mono-layered epithelium in the areas treated with Comano thermal water; inflammatory cells are numerous both in the areas treated with petrolatum gauze alone and with saline solution while such features are far less in the areas treated with Comano thermal water. Typical histology of rabbit normal skin is shown in Fig. 7.

Day 4. In the areas treated with petrolatum gauze alone a persistent abundant inflammatory infiltrate could be displayed 
Table III. Semi-quantitative assessment of the regenerated epithelial layer features and of the extent of the inflammatory infiltrate in differently treated skin graft donor sites at Days 3 and 4 in the second step of the trial.

\begin{tabular}{|c|c|c|c|c|}
\hline \multirow[t]{2}{*}{ Area } & \multicolumn{2}{|c|}{ Day 3} & \multicolumn{2}{|c|}{ Day 4} \\
\hline & Epithelium & Infiltrate & Epithelium & Infiltrate \\
\hline \multirow[t]{8}{*}{ A, Petrolatum alone } & 0 & 2 & 1 & 2 \\
\hline & 0 & 2 & 1 & 2 \\
\hline & 0 & 2 & 1 & 2 \\
\hline & 0 & 3 & 0 & 3 \\
\hline & 0 & 3 & 0 & 3 \\
\hline & 0 & 3 & 0 & 3 \\
\hline & 0 & 2 & 1 & 2 \\
\hline & 0 & 2 & 1 & 2 \\
\hline \multirow[t]{8}{*}{ B, Saline solution } & 1 & 2 & 2 & 1 \\
\hline & 1 & 2 & 2 & 1 \\
\hline & 1 & 2 & 2 & 1 \\
\hline & 1 & 3 & 1 & 2 \\
\hline & 1 & 3 & 1 & 2 \\
\hline & 1 & 3 & 1 & 2 \\
\hline & 1 & 2 & 2 & 1 \\
\hline & 1 & 2 & 2 & 1 \\
\hline \multirow[t]{8}{*}{$\mathrm{C}$, Comano water } & 2 & 1 & 3 & 0 \\
\hline & 2 & 1 & 3 & 0 \\
\hline & 2 & 1 & 3 & 0 \\
\hline & 2 & 2 & 3 & 1 \\
\hline & 2 & 2 & 3 & 1 \\
\hline & 2 & 2 & 3 & 1 \\
\hline & 2 & 2 & 3 & 1 \\
\hline & 2 & 1 & 3 & 0 \\
\hline \multirow[t]{8}{*}{$\mathrm{D}$, Untreated skin } & 3 & 0 & 3 & 0 \\
\hline & 3 & 0 & 3 & 0 \\
\hline & 3 & 0 & 3 & 0 \\
\hline & 3 & 0 & 3 & 0 \\
\hline & 3 & 0 & 3 & 0 \\
\hline & 3 & 0 & 3 & 0 \\
\hline & 3 & 0 & 3 & 0 \\
\hline & 3 & 0 & 3 & 0 \\
\hline
\end{tabular}

in all biopsies while some isolated epithelial cells appeared in a few samples (Fig. 8).

In the areas treated with sterile saline solution, a continuous mono-layered epithelium was demonstrated in most of the of samples (Fig. 9); inflammatory cells were fairly less than in the areas treated with petrolatum gauze alone. In the areas treated with Comano thermal water, all of the areas had healed, with a regenerated multilayered epithelium and just few to nil inflammatory cells; no signs of oedema could be observed (Fig. 10).

\section{Statistical analysis}

Re-epithelialisation. Table IV summarizes the trend of epithelialisation scores among the different treatments. In all animals treated with Comano thermal water $(n=8)$ the epithelialisation score increased $(n=8 / 8,100 \% ; P=0.003)$. Particularly, all of the animals were scored $2(n=8,100 \%)$ on Day 3 and scored 3 on Day $4(n=8,100 \%$ from score 2 to score 3$)$.

The epithelialisation score was increased in only $62.5 \%$ of the animals treated with saline solution $(\mathrm{N}=5 / 8,62.5 \% ; \mathrm{P}=0.018)$. Particularly, all of the animals were scored 1 on Day $3(n=8$, $100 \%)$ and 5 animals were scored 2 on Day $4(n=5,62.5 \%$; from score 1 to score 2). Similarly in the group of animals treated with petrolatum gauze $(n=8)$ the epithelialisation score was increased only in $62.5 \%$ of the animals $(\mathrm{N}=5 / 8,62.5 \%$; $\mathrm{P}=0.018)$. Particularly, all of the animals were scored 0 on Day $3(n=8,100 \%)$ and just 5 animals were scored 1 on Day 4 $(\mathrm{n}=5,62.5 \%$; from score 0 to score 1$)$. 

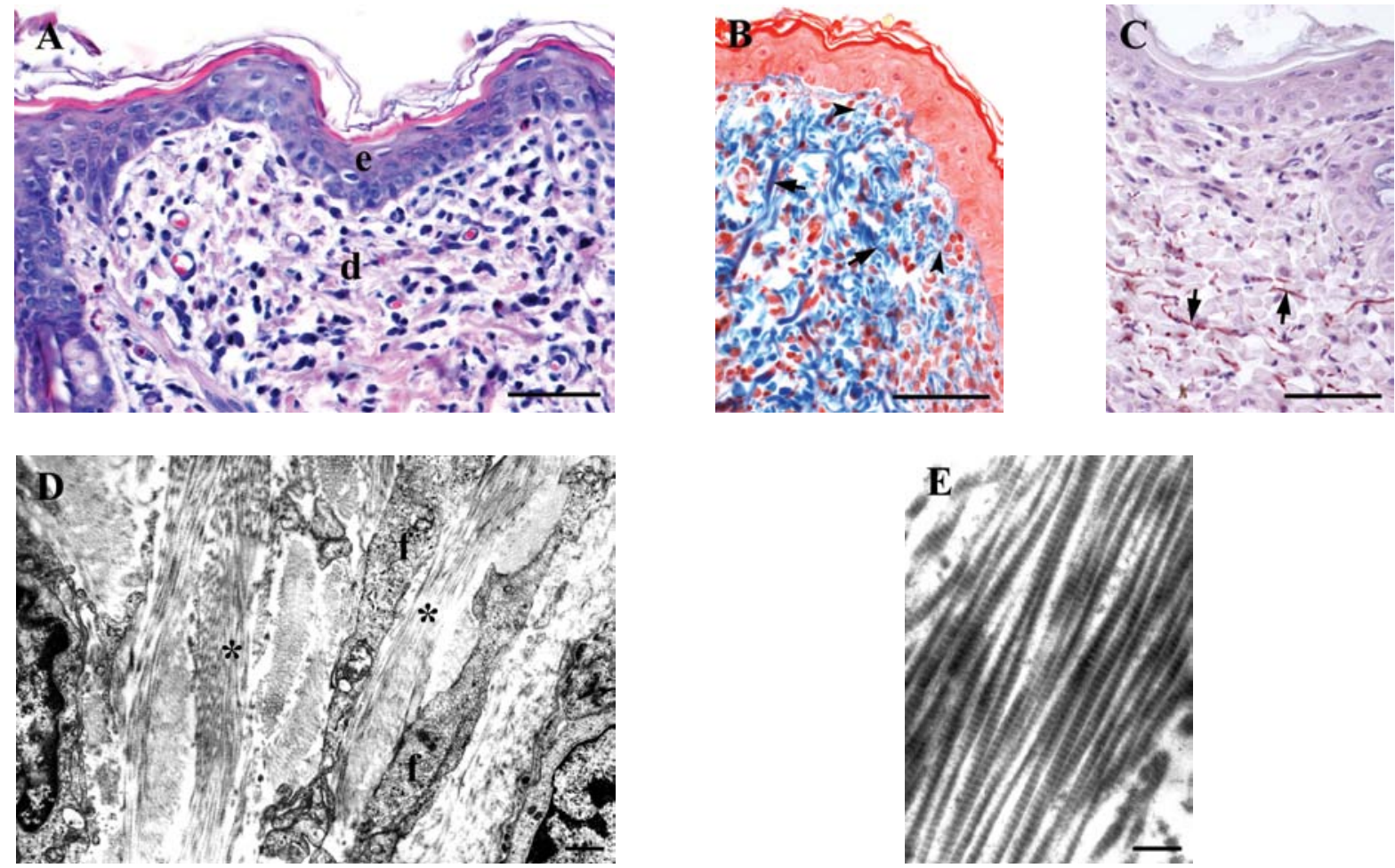

Figure 7. Rabbit normal skin. (A) Light microscopy, haematoxilin and eosin staining. Bar, $50 \mu \mathrm{m}$. The epithelium is multilayered but thin (e); the dermis (d) contains just few leucocytes. (B) Light microscopy, Masson's trichrome staining; collagen is blue-coloured. Bar, $50 \mu \mathrm{m}$. Subtle reticular fibrils can be observed at the dermo-epidermal junction (arrows) while in the dermis many bundles of reciprocally-braided collagen fibres are well evident (arrowheads). (C) Light microscopy, orcein staining; elastin looks brown-coloured. Bar, $50 \mu \mathrm{m}$. In the dermis there are numerous elastic fibres, some of them parallel to the epidermis (arrows). (D) Transmission electronic micrograph. Bar, $1 \mu \mathrm{m}$. In the dermis there are numerous bundles of mature collagen fibres (asterisks) and fibroblasts (f). (E) Transmission electronic micrograph. Bar, $0.5 \mu \mathrm{m}$. The high magnification points out the regularity of the collagen fibres.
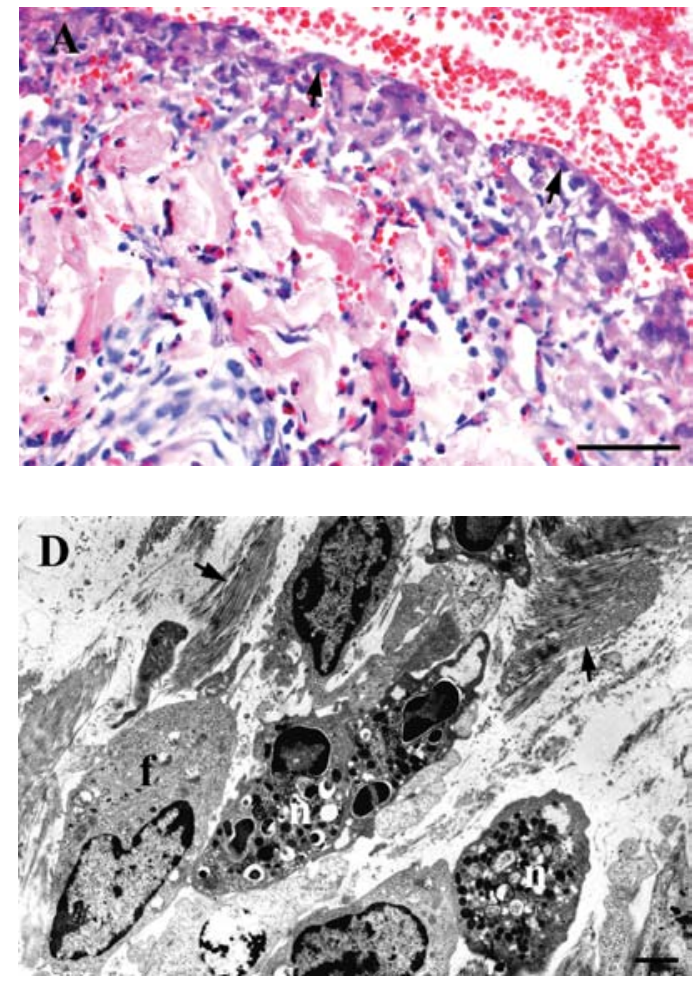
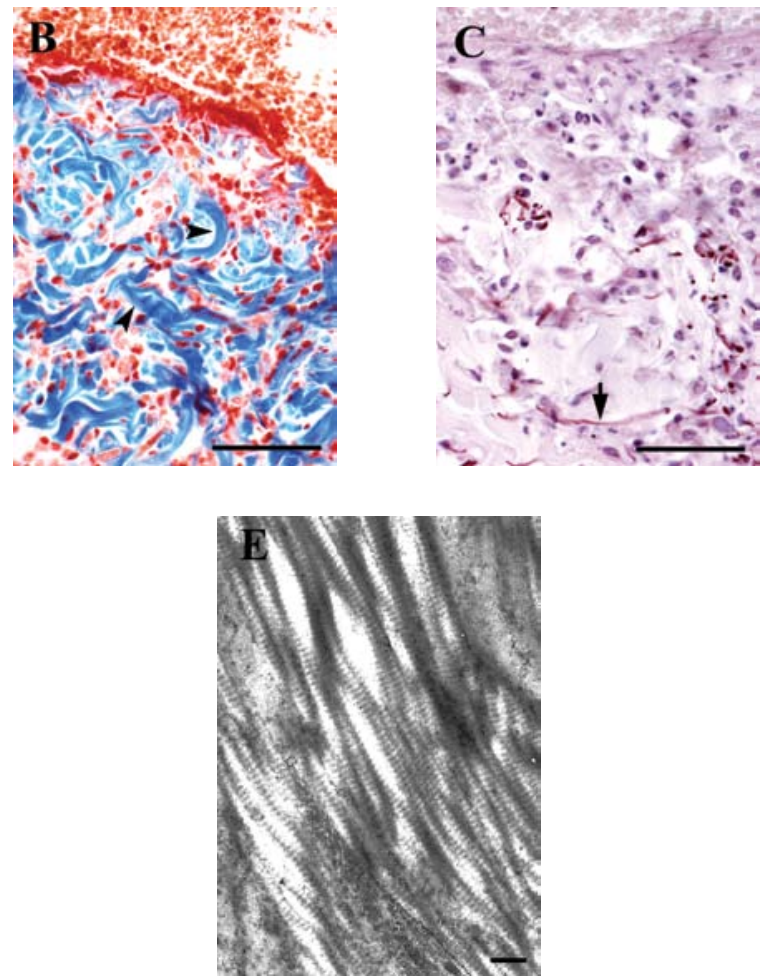

Figure 8. Rabbit skin graft donor site dressed with petrolatum gauze 4 days after graft harvesting. (A) Light microscopy, haematoxilin and eosin staining. Bar, $50 \mu \mathrm{m}$. Just few scattered epithelial cells (arrows) and a fair inflammatory infiltrate in the dermis are observed. (B) Light microscopy, Masson's trichrome staining; collagen is blue-coloured. Bar, $50 \mu \mathrm{m}$. The collagen in the dermis is arranged in thick bundles of fibres. (C) Light microscopy, orcein staining; elastin is brown-coloured. Bar, $50 \mu \mathrm{m}$. Elastic fibres look fragmented and just a few of them are arranged parallel to the epidermis (arrows). (D) Transmission electronic micrograph. Bar, $4 \mu \mathrm{m}$. Disorganised bundles of collagen fibres (arrows) are observed among fibroblasts (f) and neutrophils in great number (n). (E) Transmission electronic micrograph Bar, $0.5 \mu \mathrm{m}$. The disorganization of collagen is proven by the spaces between the fibres. 

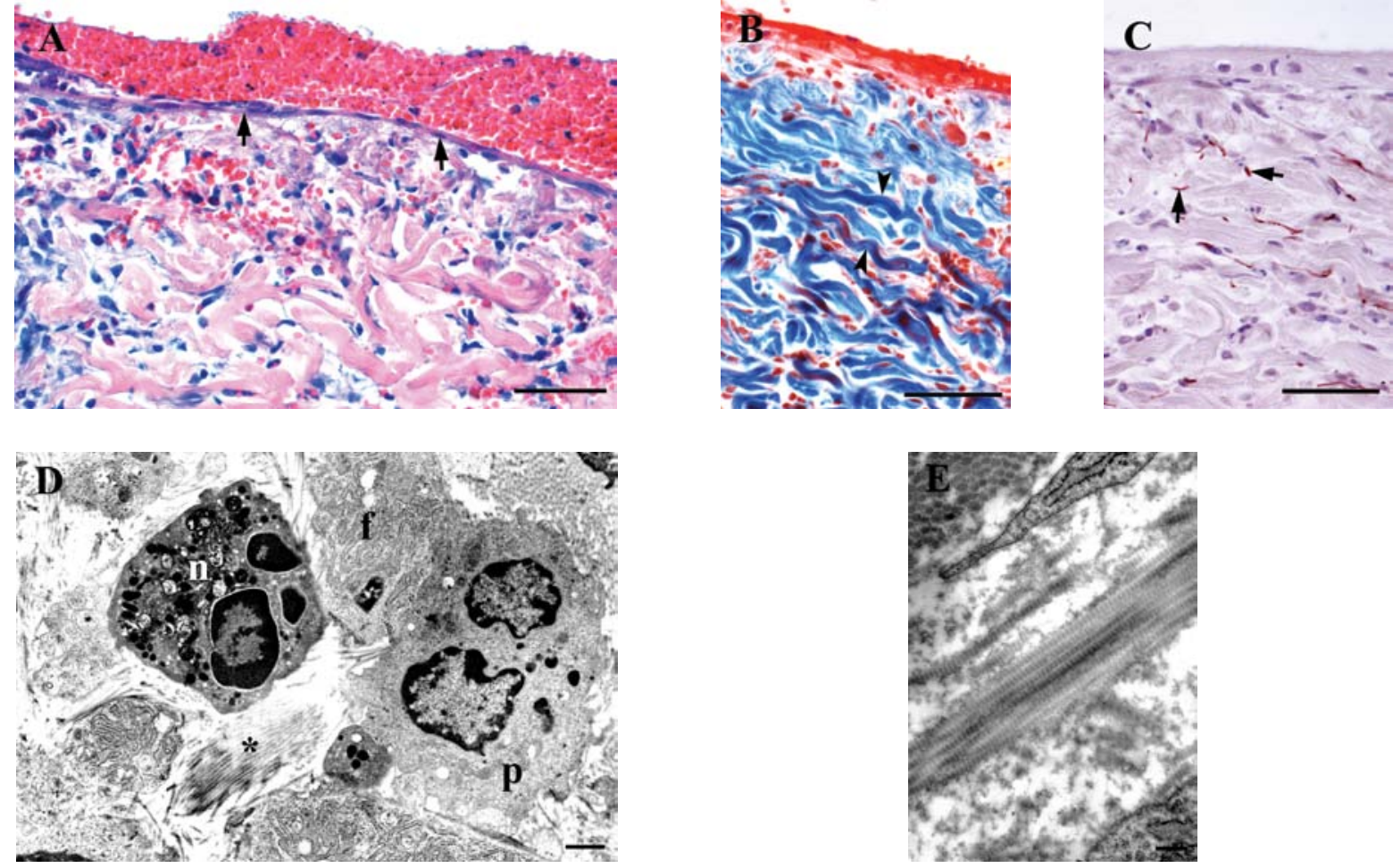

Figure 9. Rabbit skin graft donor site dressed with sterile saline solution 4 days after graft harvesting. (A) Light microscopy, haematoxilin and eosin staining. Bar, $50 \mu \mathrm{m}$. The epidermis consists of a single layer of epithelial cells (arrows) under the residuals of a bloody scab. Inflammatory cells are still observed in the dermis. (B) Light microscopy, Masson's trichrome staining; collagen is blue-coloured Bar, $50 \mu \mathrm{m}$. The collagen fibres are mostly gathered in thick bundles (arrowheads). (C) Light microscopy, orcein staining; elastin is brown-coloured. Bar, $50 \mu \mathrm{m}$. Elastic fibres look fragmented (arrows). (D) Transmission electronic micrograph. Bar, $4 \mu \mathrm{m}$. Neutrophil granulocytes (n), plasmacells (p) and fibroblasts (f) are observed among thin bundles of collagen fibres. (E) Transmission electronic micrograph. Bar, $0.5 \mu \mathrm{m}$. The bundles of collagen fibres look thin and regular.
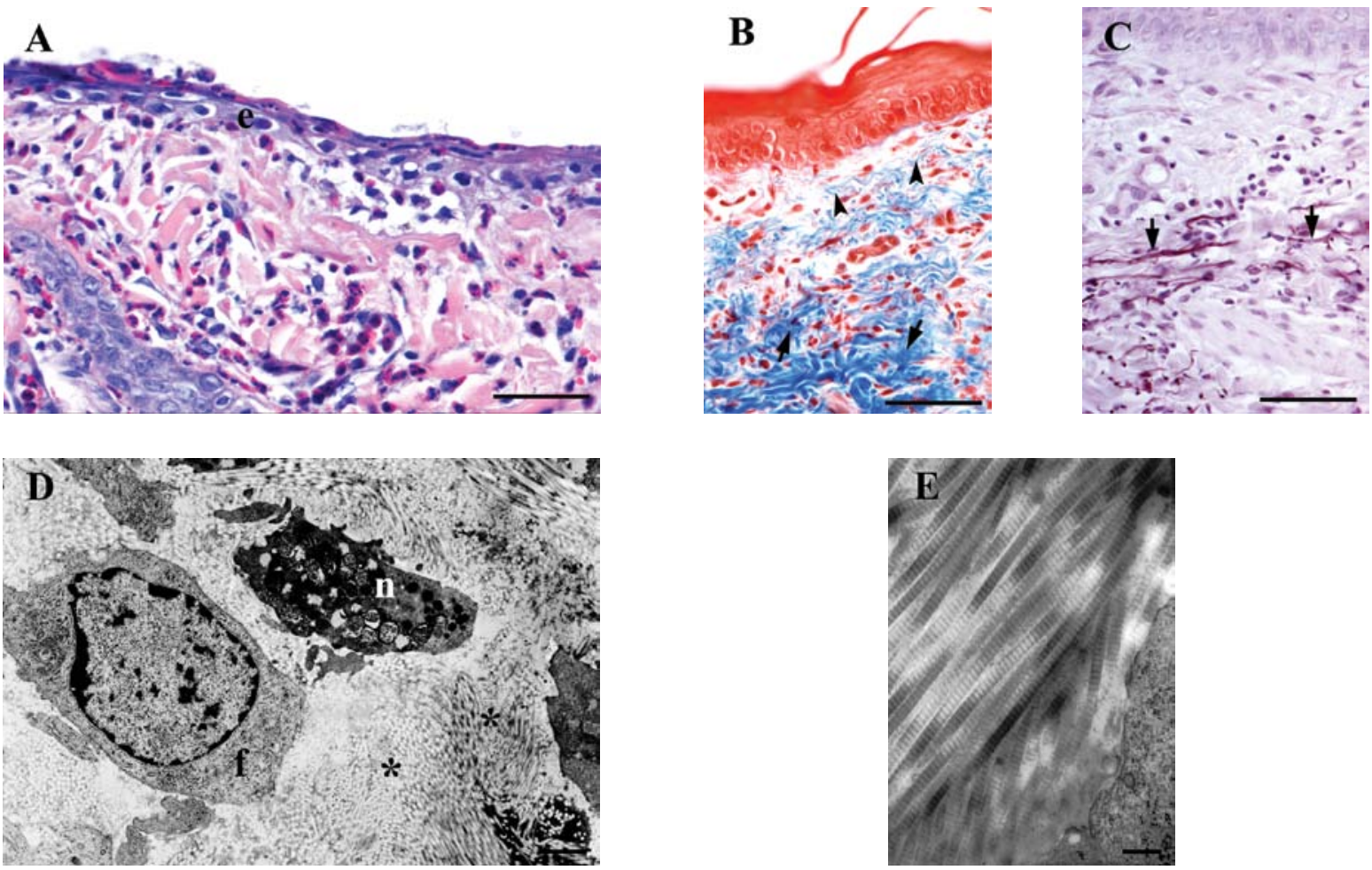

Figure 10. Rabbit skin graft donor site dressed with Comano thermal water 4 days after graft harvesting. (A) Light microscopy, haematoxilin and eosin staining. Bar, $50 \mu \mathrm{m}$. The epidermis looks multilayered (e); just a few inflammatory cells infiltrate the dermis. (B) Light microscopy, Masson's trichrome staining; collagen is blue coloured. Bar, $50 \mu \mathrm{m}$. A fine network of collagen fibres is restored at the dermo-epidermal junction (arrowheads.) The bundles of collagen fibres in the dermis have a normal size (arrows.) (C) Light microscopy, orcein staining; elastin is brown-coloured. Bar, $50 \mu \mathrm{m}$. The elastic fibres show a length and a direction similar to the ones in normal skin (arrows). (D) Transmission electronic micrograph. Bar, $3 \mu \mathrm{m}$. In the dermis, bundles of well-organised collagen fibres (asterisks) are observed among fibroblasts (f) and a few neutrophils (n). (E) Transmission electronic micrograph. Bar, $0.5 \mu \mathrm{m}$. The high magnification points out the regularity of the collagen fibres. 
Table IV. Epithelialisation score observed between the 3rd and the 4th day, depending on the different treatments.

Epithelization score

\begin{tabular}{lcccr}
\cline { 2 - 4 } Treatment & $\begin{array}{c}\text { Increased } \\
\mathrm{N}(\%)\end{array}$ & $\begin{array}{c}\text { Unchanged } \\
\mathrm{N}(\%)\end{array}$ & $\begin{array}{c}\text { Decreased } \\
\mathrm{N}(\%)\end{array}$ & \begin{tabular}{c} 
P-value \\
\hline Petrolatum gauze
\end{tabular} \\
Saline solution & $5(62.5)$ & $3(37.5)$ & $0(0)$ & 0.018 \\
Comano thermal water & $5(62.5)$ & $3(37.5)$ & $0(0)$ & 0.018 \\
Untreated skin & $8(100)$ & $0(0)$ & $0(0)$ & 0.003 \\
\hline
\end{tabular}

Table V. Inflammatory infiltrate score observed between the 3rd and the 4th day, depending on the different treatments.

\begin{tabular}{|c|c|c|c|c|}
\hline \multirow[b]{2}{*}{ Treatment } & \multicolumn{3}{|c|}{ Inflammatory infiltrate score } & \multirow[b]{2}{*}{ P-value } \\
\hline & $\begin{array}{c}\text { Increased } \\
\mathrm{N}(\%)\end{array}$ & $\begin{array}{l}\text { Unchanged } \\
\mathrm{N}(\%)\end{array}$ & $\begin{array}{c}\text { Decreased } \\
\mathrm{N}(\%)\end{array}$ & \\
\hline Petrolatum gauze & $0(0)$ & $8(100)$ & $0(0)$ & 1 \\
\hline Saline solution & $0(0)$ & $0(0)$ & $8(100)$ & 0.003 \\
\hline Comano thermal water & $0(0)$ & $0(0)$ & $8(100)$ & 0.003 \\
\hline Untreated skin & $0(0)$ & $8(100)$ & $0(0)$ & 1 \\
\hline
\end{tabular}

Inflammatory infiltrate. Table $\mathrm{V}$ summarizes the trend of the inflammatory infiltrate scores among the different treatments. The inflammatory infiltrate score was decreased in all of the animals treated with Comano thermal water $(n=8 / 8,100 \%$, $\mathrm{P}=0.003)$. Particularly, on Day 3, 4 animals were scored 2 $(n=4,50 \%)$ and the same were scored 0 on Day 4 (from score 1 to score 0 ); the others 4 animals were scored 1 on Days 3 and scored 2 on Day 4 (from score 2 to score 1). The inflammatory infiltrate score was decreased in all of the animals treated with saline solution $(n=8 / 8,100 \% ; P=0.003)$. Particularly, 3 animals were scored 3 on Day $3(n=3,37.5 \%)$ and the same were scored 2 on Day 4 (from score 3 to score 2); the others 5 animals $(n=5,62.5 \%)$ were scored 2 on Days 3 and 1 on Day 4 (from score 2 to score 1 ). The inflammatory infiltrate score was not decreased in any of the animals treated with petrolatum gauze $(n=8)$. Particularly, on Day 3, 3 animals $(37.5 \%)$ were scored 3 , while the others 5 animals were scored 2. On Day 4 in all of the animals the score was unchanged.

Masson's trichrome and TEM analysis. Findings from the samples stained with Masson's trichrome and orcein and under TEM were not subjected to statistical analysis. In the dermis of the areas treated with petrolatum gauze alone, the collagen fibres stained with Masson's dye appeared thicker and with an oblique pattern if compared with those of the untreated skin; with transmission electronic microscope (TEM), they looked irregular and separated by large spaces filled by many neutrophil granulocytes.

Also in the areas treated with sterile saline solution the collagen fibres stained with Masson's dye looked thicker than in the untreated skin but showed a higher density than in the areas treated with petrolatum gauze alone; with TEM, they appeared in an advanced phase of reorganisation with an intense ongoing polymerisation process including some plasmacells in the field. In the areas treated with Comano thermal water the collagen fibres stained with Masson's dye showed a diameter similar to those in the normal skin and were arranged in a more homogeneous pattern than in the areas treated with petrolatum gauze or with sterile saline solution; at the TEM, they appeared longitudinally oriented and well structured and just rare granulocytes were detectable in the dermis.

The elastic fibres (stained with orcein) both present in the areas treated with petrolatum gauze alone and in the areas treated with sterile saline solution looked shorter than in the untreated skin; on the contrary, in the areas treated with Comano thermal water an overall higher density in the elastic dermal network was detectable, with longer elastic fibres than in the other areas and with a pattern similar to the normal skin.

\section{Discussion}

Keratinocytes continue to reproduce through the whole lifetime and such an activity has been shown to decrease in many clinical situations, such as difficult-to-heal wounds, chrono-aging and photo-aging. An increasing number of studies attempting to identify topical substances and effective methods to improve safe epithelial regeneration have been reported in the literature.

Thermal mineral water treatments have been demonstrated to promote healing of skin irritations (5) and to improve skin restoration after cosmetic aggressive procedures $(6,7)$. Such effects are likely to be related to some anti-inflammatory action $(8,9)$. It is also well demonstrated that the proliferative phase of wound healing is inversely proportional to the amount of inflammatory post-traumatic reaction (10). 
Following these preliminary considerations, the basic assumptions that founded this trial were: i) the evidence that wound healing is favoured by a wet environment (11) and ii) the proven anti-inflammatory properties of Comano thermal water (1-3). In order to avoid any bias related to a clinical observational human study, the putative activity of Comano thermal water on skin regeneration was tested in a standard animal experimental model. Split thickness skin graft donor sites represented an ideal experimental wound model to assess keratinocyte regeneration following different treatments; in such a site a spontaneous re-epithelialisation is known to occur within 2 to 3 weeks in humans by means of an active cell proliferation from the remaining epidermal appendages located in the deep reticular dermis.

In current clinical practice a split thickness skin graft donor site is covered with an occlusive local dressing that is left in place until re-epithelialisation allows its spontaneous removal. A petrolatum gauze covered with a layer of non-woven fabric gauzes is one of the most popular occlusive dressing for a split thickness skin graft donor site. In order to balance the potential prejudicial advantage of the wet Comano thermal water dressing over the non-wet traditional petrolatum gauze dressing (12) in the experimental wound, we decided to include in the test a different kind of wet dressing, the saline solution.

The results of our experience were uniform throughout the whole sample with the clinical pictures exactly overlapping the histological observations. Such figures consistently demonstrated a strong efficacy of Comano thermal water in promoting the skin healing process. Major differences among the different treatments were highlighted on Days 3 and 4 after the trauma, as demonstrated by the number and location of the regenerated keratinocytes and the number of inflammatory cells (neutrophil granulocytes) in the field.

As the wound healing pathway is shorter in animals than in humans (13), the overall wound healing time was anticipated in all sites. In such a context the areas treated with petrolatum gauze healed in 6 days; at this time the histological figure showed a persistent rich inflammatory reaction, in the absence of any sign of infection.

The areas treated with saline solution, as expected, healed in 5 days and showed a less important inflammatory reaction. The areas treated with Comano thermal water healed faster than any other site: complete clinical healing occurred in just 4 days at which time the histological examination demonstrated a multilayered regenerated epithelium with an almost absent inflammatory reaction. Interestingly, if not surprisingly, some important architectural differences in the dermis of differently treated areas could also be appreciated.

On Day 4 after trauma, the areas treated with Comano thermal water were the only sites showing collagen fibres almost normal in shape, size and pattern. The elastic fibres network also showed a pattern similar to the normal skin with longer elastic fibres than in the other areas. It is well known that epithelial and dermal regenerations are closely related, with a reciprocal influence (14).

A possible explanation for the excellent results of the topical treatment with Comano thermal water, could be a synergistic enhancement of the wound healing process by the favourable combination of a local wet environment with the anti-inflammatory action. Actually, some studies have shown that Comano thermal water is able to reduce the expression of some cytokines (TNF- $\alpha$, IL-6 and IL-8) involved in the inflammatory process, relating this property to its anti-psoriatic efficacy in humans $(15,16)$. Unlike corticosteroid action, such a specific effect against the inflammatory reaction does not counteract cell proliferation. Following our experience, the regenerative properties of Comano thermal water observed in rabbits could be applied for humans use.

\section{Acknowledgements}

We are indebted to Mr. GianMario Pelizzoli, technician at the University of Pavia, for his much appreciated contribution to the management of the digital pictures, to Dr Alberto Malovini, statistician on duty at the IRCCS Fondazione 'S. Maugeri', Pavia, for the statistical analysis and to Centro di ricerca e studio dell' Azienda Consorziale 'Terme di Comano' (Trentino, Italy) for financial support.

\section{References}

1. Zumiani G, Zanoni M, Lo Brutto G, Cristofolini M and Tasin L: Bathphoto-therapy with the thermal water of Comano: treatment of psoriasis. Acta Derm Venereol Suppl (Stockh) 146: 122-124, 1989.

2. Zumiani G, Zanoni M and Agostini G: Valutazione dell'efficacia dell'acqua della fonte termale di Comano versus acqua di acquedotto nella cura della psoriasi. G It Dermatol Venereol 135: 259-263, 2000 (In Italian).

3. Psoriasi e terapia termale a Comano. http://www.termecomano. it/documenti/AreaMedici/2009.

4. R Development Core Team: R: A language and environment for statistical computing. R Foundation for Statistical Computing. Vienna, 2009.

5. Ferreira MO, Costa PC and Bahia MF: Effect of São Pedro do Sul thermal water on skin irritation. Int J Cosmet Sci 32: 205-210, 2010.

6. Goldman MP, Merial-Kieny C,Nocera T and Mery S: Comparative benefit of two thermal spring waters after photodynamic therapy procedure. J Cosmet Dermatol 6: 31-35, 2007.

7. Barolet D, Lussier I, Mery S and Merial-Kieny C: Beneficial effects of spraying low mineral content thermal spring water after fractional photothermolysis in patients with dermal melasma. $\mathbf{J}$ Cosmet Dermatol 8: 114-118, 2009.

8. Castex-Rizzi N, Charveron M and Merial-Kieny C: Inhibition of TNF-alpha induced-adhesion molecules by Avène Thermal Spring Water in human endothelial cells. J Eur Acad Dermatol Venereol 25 (Suppl 1): S6-S11, 2011.

9. Ghersetich I and Lotti T: Immunologic aspects: immunology of mineral water spas. Clin Dermatol 14: 563-566, 1996.

10. Werner S and Grose R: Regulation of wound healing by growth factors and cytokines. Physiol Rev 83: 835-870, 2003.

11. Bolton L: Operational definition of moist wound healing. J Wound Ostomy Continence Nurs 34: 23-29, 2007.

12. Persson K and Sålemark L: How to dress donor site of split thickness skin grafts: a prospective, randomized study of four dressings. Scand J Plast Reconstr Surg Hand Surg 34: 55-59, 2000.

13. Gurtner G, Werner S, Barrandon Y and Longaker MT: Wound repair and regeneration. Nature 453: 314-321, 2008.

14. Werner S, Krieg T and Smola H: Keratinocyte-fibroblast interactions in wound healing. J Invest Dermatol 127: 998-1008, 2007.

15. Chiarini A, Dal Pra I, Pacchiana R, Menapace L, Zumiani G, Zanoni M and Armato U: Comano's (Trentino) thermal water interferes with the expression and secretion of vascular endothelial growth factor-A protein isoforms by cultured human psoriatic keratinocytes: A potential mechanism of its anti-psoriatic action. Int J Mol Med 18: 17-25, 2006.

16. Dal Pra I, Chiarini A, Pacchiana R, Zumiani G, Zanoni M and Armato U: Comano's (Trentino) thermal water interferes with tumour necrosis factor-alpha expression and interleukin-8 production and secretion by cultured human psoriatic keratinocytes: Yet other mechanisms of its anti-psoriatic action. Int J Mol Med 19: 373-379, 2007. 